\title{
A comparison study of two multifidelity methods for aerodynamic optimization
}

\author{
Spyridon G. Kontogianis ${ }^{1}$, Jean Demange ${ }^{1}$, A. Mark Savill ${ }^{2}$, Timoleon Kipouros ${ }^{2}$ \\ Cranfield University, Bedfordshire, MK430AL, United Kingdom
}

\begin{abstract}
Industrial aerodynamic design applications require multiobjective optimization tools able to provide design feedback to the engineers. This is true especially when optimization studies are carried out during the conceptual design stage. The need for fast optimization methods has led to the development of multifidelity methods in a surrogate based optimization environment. Multifidelity tools have the potential to accelerate the design process, primarily due to the lower cost associated with the low fidelity tool. In addition to this, the design stage is shortened as mature and reliable high fidelity design information is provided earlier in the design cycle. Despite this high potential of these methods, there is no explicit comparison available in the literature between multifidelity surrogate based optimization tools for industrial aerodynamic problems. This paper aims at providing a direct comparison between two multiobjective multifidelity surrogate based optimization methods developed by our group. The first approach uses a trust region formulation for efficient multiobjective that does not require gradients. The second is using the concept of expected improvement to perform fast design space exploration based on a novel Kriging modification for multifidelity data. The tools are applied in two aerodynamic design problems: optimization of a high lift configuration in respect to maximum lift maximization and an airfoil design for transonic cruising conditions. These problems feature characteristics of industrial interest. They involve difficult physical analyses in the case of the high lift configuration and a more complex optimization formulation due to the increased dimensionality in the case of the transonic airfoil. Our presented methods are compared against a CFD-based optimization, a surrogate based optimization using only high fidelity data and a multifidelity surrogate based optimization based on Co-Kriging. Early results suggest that the trust region method can quickly
\end{abstract}


provide improved designs leading to an efficient pareto front. The expected improvement based method shows fast exploration attributes and a wide pareto front.

\section{Introduction}

NDUSTRIAL applications of aerodynamic design demand the use of tools capable of Igenerating efficient designs in a series of objectives. In the initial stages of the design, perhaps the most critical attribute of an optimization tool would be the ability to provide

5 the maximum amount of information to the designer, thus acting as a very efficient trade study tool. Equally important, the tool should be able to provide reliable assessment of the concepts considered, even at these early design stages. This can be critical for a success of the new design concept as it shortens the design cycle.

To do so, such an optimization framework should be fast, minimizing any cost associated with guiding the optimizer, as well as the cost of the analysis itself. In the center of this lies the surrogate-based optimization (SBO) and the multifidelity (MF) methods.

However, to take full advantage of these techniques and to provide more information and physical insight according to the aforementioned, SBO and MF methods should be used within a multiobjective (MO) formulation.

Although MO optimization methods have been used in the aerospace industry [1, 2], tools based solely on gradient-free MO optimizers might lead to very high computational expenses [3]. In response to that, there has been an effort to reduce the cost by using surrogate modelling and multifidelity [4] techniques, which have a profound effect when the metamodels substitute expensive [5] or challenging analyses. Their use is not limited in decreasing the computational requirements associated with high fidelity (HF) analysis but to guide the design [6] as well, as in SBO problems. Typical applications of metamodeling-based methods involve airfoil or wing design $[7,8]$, aeronautical and aerospace applications $[9,10,11,12]$. Effort to develop more sophisticated metamodeling techniques focus on the accuracy and flexibility $[13,14]$ of the models. Another

\footnotetext{
${ }^{1}$ School of Aerospace, Transport and Manufacturing, department of Power \& Propulsion.

${ }^{2}$ Professor of Computational Aerodynamics Design, School of Aerospace, Transport and Manufacturing, Propulsion Engineering Centre.

${ }^{3}$ Research Fellow, School of Aerospace, Transport and Manufacturing, Propulsion Engineering Centre. Preprint submitted to Aerospace Science and Technology 
approach towards better exploitation of metamodeling for optimization is the use of multifidelity analysis, as industries use analyses of different fidelities depending on the design stage [15]. Since optimization methods are not acting on the analysis but on a surrogate generated by the analyses' data, using data of variable fidelity tools is a sensible way to accelerate a design problem associated with an expensive analysis as well as being used to steer to designs more insensitive to operating conditions [16]. Popular formulations based on this have have been developed $[17,18,19,20]$ and applied $[21,22,23,24]$ in the recent years.

Following the needs outlined in the beginning of the introduction, our group has been involved in the development of two distinctive methodologies employing MF SBO techniques in an MO formulation. The first one is a trust region (TR)-based frame30 work [25] that uses a metamodel to correct the low fidelity (LF) aerodynamic solver, steering the method to high fidelity optimality. The method does not require gradients information [26, 27] nor an initial sampling. The second approach [28] is based on a multifidelity variation of the Kriging metamodel, applied within the global surrogatebased optimization strategy using the expected improvement (EI) [29] criterion. Both methods can tackle multiobjective problems efficiently with the multiobjective optimization acting directly on very cheap function calls. The multiobjective optimizer used is an inhouse implementation of the multiobjective particle swarm optimization (MOPSO) algorithm [30].

This paper provides a direct and systematic comparison of the above methods, aiming to display the appropriateness of each approach and their dependency on the problem at hand. To validate and further assess these methods, our results are also compared against a CFD-based gradient-free method, a high fidelity SBO method using EI criterion and a MF EI method using Co-Kriging to handle the MF data. Two typical industrial aerodynamic design problems are used as test cases: The first design problem involves the take-off performance maximization, translated as a $c_{l_{\max }}$ and lift-over-drag ratio maximizations, of the Garteur high lift three elements configuration [31, 32]. This

45 is a problem involving six design variables and a challenging aerodynamic analysis problem. The second design scenario resembles a typical airfoil design for transonic cruising conditions using RAE2822 airfoil [28]. Here, $C_{l}$ and $C_{d}$ are used as objectives. 
In section 2, the two methodologies are described in more detail. Section 3 provides an overview of the formulation of each aerodynamic optimization case. The results of each methodology at each test case are shown in Section 4. A summary outlining the conclusions extracted by this work as well as the further future work is provided in Section 5 .

\section{2. Methodology}

Let $f_{\text {low }}: \mathbb{R}^{n} \rightarrow \mathbb{R}^{l}$ be the low-fidelity function and $\boldsymbol{f}_{\text {high }}: \mathbb{R}^{n} \rightarrow \mathbb{R}^{l}$ be the highfidelity function; where $n \geq 1$ is the design space size and $l \geq 1$ is the objective space size. The goal is the minimization (or maximization) of the high-fidelity function as defined in equation (1), where $\boldsymbol{x}$ is a vector of dimension $n$ and $\mathcal{D}$ is a subset of $\mathbb{R}^{n}$ :

$$
\min _{\boldsymbol{x} \in \mathcal{D}} f_{\text {high }}(\boldsymbol{x})
$$

\section{2.1. Trust-Region-Based}

With this method, the problem (1) is solved by a derivative-free trust region method presented by Conn et al. [26]. Instead of directly solving the high-fidelity problem, an approximation model is used, and at iteration $k$, the problem (2) is solved under a trust region $\mathcal{T}_{k}$ :

$$
\min _{\boldsymbol{x} \in \mathcal{T}_{k}} \boldsymbol{m}_{k}(\boldsymbol{x}) .
$$

${ }_{60}$ The trust region is usually defined as a ball centered on the initial point of the subproblem but in Ref. [33], a second definition based on the number of improvements of the corrected model is used. This paper only uses the step-based trust region presented in more details in section 2.1.3.

The model $\boldsymbol{m}_{k}: \mathcal{T}_{k} \rightarrow \mathbb{R}^{l}$ is defined by (3) at iteration $k$. It is formed by the sum of a Kriging surrogate model $\boldsymbol{e}_{k}$ representing the difference between the low- and the high-fidelity functions (the error in other terms) and of the low-fidelity function.

$$
\boldsymbol{m}_{k}(\boldsymbol{x})=\boldsymbol{f}_{\text {low }}(\boldsymbol{x})+\boldsymbol{e}_{k}(\boldsymbol{x})
$$

It was shown in reference [25] that using a RBF or Kriging model was not enough to reach the same Pareto front extend than compared to the high-fidelity-only optimisation. 
Therefore, in this work, a co-Kriging model is used when a sufficient number of points is available for its training. Hence, when more than $2(n+1)$ high-fidelity points are available for training, the co-Kriging model is built and equation (3) is changed for equation (4):

$$
\boldsymbol{m}_{k}(\boldsymbol{x})=\boldsymbol{e}_{k}(\boldsymbol{x})
$$

with $\boldsymbol{e}_{k}(\boldsymbol{x})$ the co-Kriging model, implemented according to Forrester et al [34, 35]. A co-Kriging model extends the original Kriging model to include multiple level of fidelity 70 of the simulation. Therefore in this case, the model is built with high- and low-fidelity points, and the co-Kriging model no longer predicts the error but predicts directly the corrected function.

A summary of the method is presented in the flowchart 1 .

\subsubsection{Subproblem Optimizer}

To solve the problem (2), the MultiObjective Tabu Search [36, 37] (MOTS) optimizer 75 is used. The local search is handled by a Hooke and Jeeves move, choosing the best direction to move by comparing improvements in each direction. To avoid being trapped in local minima, MOTS records all the points that have been visited, forbids their reuse, which in turn provides the ability to climb away from local minima to explore the design space. Memories are also used to record design space portions that have been visited,

so a characteristic which is eventually exploited when no more improvements are found to diversify. Hence MOTS starts by a local search but include globality search with the use of memories and diversification moves.

\subsubsection{Computation of the ratio of improvement}

As for traditional trust-region algorithms, the ratio of improvements $\boldsymbol{\rho}_{k}=\left[\rho_{k}^{(1)}\right.$, $\left.\rho_{k}^{(2)}, \ldots, \rho_{k}^{(l)}\right]$ are calculated for each point from the suboptimizer and each objective as 85 follows:

$$
\rho_{k}^{(i)}=\frac{f_{\text {high }}^{(i)}\left(\boldsymbol{x}_{k}\right)-f_{\text {high }}^{(i)}\left(\boldsymbol{x}_{s}\right)}{m_{k}^{(i)}\left(\boldsymbol{x}_{k}\right)-m_{k}^{(i)}\left(\boldsymbol{x}_{s}\right)}, \quad i \in[1, \ldots, l] .
$$

\subsubsection{Trust region management}

Since each point from the current Pareto front is evaluated with the high-fidelity function, the front is updated accordingly: some points that would be Pareto equivalent 


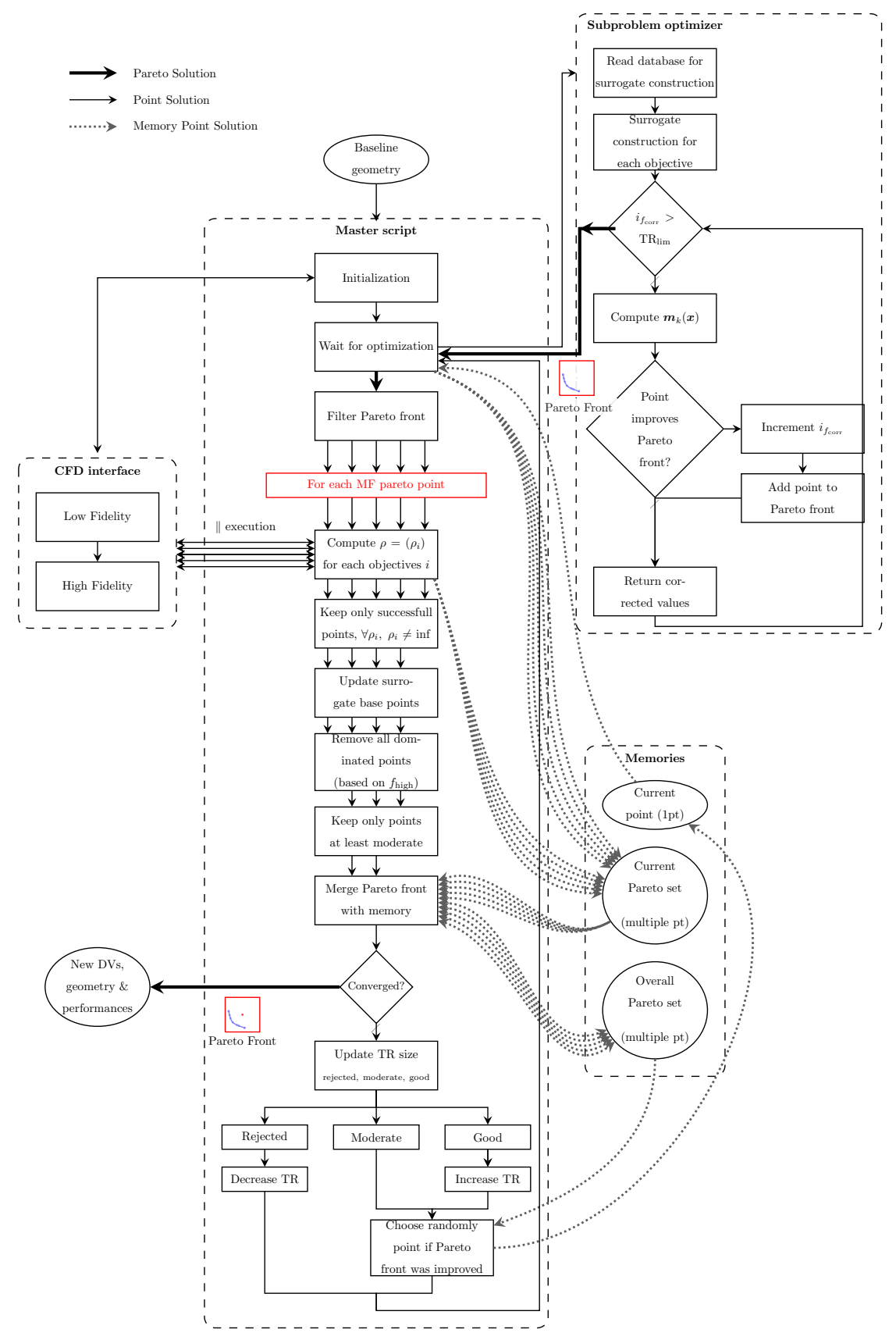

Figure 1: Multiobjective multifidelity optimization framework 
using $\boldsymbol{f}_{\text {corr }}$ won't have the same status once compared with $\boldsymbol{f}_{\text {high }}$.

The ratio of improvement's definition was extended according to equations (5) and (7) in order to be used with multiple objectives as follows:

- for each candidate point $\boldsymbol{p}_{k}=\left[f_{\text {high }}^{(1)}\left(\boldsymbol{x}_{k}\right), \ldots, f_{\text {high }}^{(l)}\left(\boldsymbol{x}_{k}\right)\right]$ in the objective space,

- if the number of objectives having their ratio of improvement greater than $\rho_{\text {bad }}$ is higher or equal to the trigger $n_{f}$, the point $\boldsymbol{p}_{k}$ is marked moderate;

- if the number of objectives having their ratio of improvement strictly greater than $\rho_{\text {good }}$ is higher or equal to the trigger $n_{f}$, the point $\boldsymbol{p}_{k}$ is marked good;

- otherwise, the point is marked bad.

- If the number of points $\boldsymbol{p}_{k}$ marked:

- moderate is greater than the trigger $n_{p}$, the overall iteration is considered moderate,

- good is greater than the trigger $n_{p}$, the overall iteration is considered good,

- otherwise the iteration is considered bad.

The trust region is increased or shrank according to the status of the current iteration as in equation (6):

$$
\Delta_{k+1}=\left\{\begin{array}{llr}
\Delta_{k}-\gamma_{s} & \left|\left\{\boldsymbol{p}_{\text {moderate }}\right\}\right|<n_{p} & \text { (bad prediction) } \\
\Delta_{k} & \left|\left\{\boldsymbol{p}_{\text {moderate }}\right\}\right| \geq n_{p} & \text { (moderate prediction) } \\
\Delta_{k}+\gamma_{e} & \left|\left\{\boldsymbol{p}_{\text {good }}\right\}\right| \geq n_{p} & \text { (good prediction) } .
\end{array}\right.
$$

\subsubsection{New point selection}

The current Pareto front is then merged with the overall one and the number of points improving the Pareto front is counted. If at least one point improves the Pareto front, a new point $\boldsymbol{x}_{s}$ is randomly chosen from the overall Pareto front, otherwise the sub-optimization is restarted from the same point, but this time, with a more accurate corrected function:

$$
\boldsymbol{x}_{k+1}=\left\{\begin{aligned}
\boldsymbol{x}_{s} \text { if }\left|\left\{\boldsymbol{p}_{\text {good }}\right\}\right| \geq n_{p} \\
\text { and } \exists \boldsymbol{p} \in\left\{\boldsymbol{p}_{\text {good }}\right\}, \\
\quad \forall \boldsymbol{p}_{0} \in\left\{\boldsymbol{p}_{\text {good }} \backslash \boldsymbol{p}\right\} \exists i \in[1, \ldots, l], f_{\text {high }}^{(i)}\left(\boldsymbol{x}_{p}\right)<f_{\text {high }}^{(i)}\left(\boldsymbol{x}_{p_{0}}\right) \\
\boldsymbol{x}_{k} \text { else, }
\end{aligned}\right.
$$


with $\boldsymbol{x}_{p}$ and $\boldsymbol{x}_{p_{0}}$ being the coordinates in the design space of the respective points $\boldsymbol{p}$ and ${ }_{105} \boldsymbol{p}_{0}$.

The random selection is biased towards low-density part of the Pareto front in order to allow its entire exploration. To do so, the Euclidean distances $d_{i}$ between each consecutive point from the Pareto front $\mathcal{P}_{\text {front }}=\left\{\boldsymbol{p}_{1}, \ldots, \boldsymbol{p}_{f}\right\}$, containing $f$ points, are computed according to equation (8):

$$
\forall i \in[1, \ldots, f-1], d_{i}=\left\|\boldsymbol{p}_{i+1}-\boldsymbol{p}_{i}\right\|,
$$

and then used as weight for the random selection.

\subsection{Expected Improvement Based}

To satisfy design space exploration and global optimality requirements of an early design stage study, this methodology is based on an explorative surrogate-based optimization plan [29]. It employs a novel metamodel approach to take advantage of the availability of multifidelity data.

\subsubsection{Multifidelity analyses}

A significant number of the analyses called by the optimization scheme are performed using an LF tool, as we aim to reduce the required computational resources. The corresponding results however are corrected since the LF tool is subject to error. LF results are related to HF ones by superimposing an error prediction as follows [34],

$$
\mathbf{f}_{\text {high }}(x)=\mathbf{f}_{\text {low }}(x)+\mathbf{e}(\mathbf{x})
$$

\subsubsection{Surrogate model}

The optimization study aims in providing fast and extensive exploration in the conceptual stage, in which The functions of interest are approximated using a surrogate model. Here, the decomposition of Eq.9 demands not only a model for the error correction $e$ but also a model for the objective function $y$ of the LF tool as well. For metamodel predictions of the $\mathbf{f}_{\text {high }}$ and $\mathbf{f}_{\text {low }}$ functions, Eq.9 becomes,

$$
\mathbf{y}_{\text {high }}(\mathbf{x})=\mathbf{y}_{\text {low }}(\mathbf{x})+\mathbf{e}(\mathbf{x})
$$

In the case of a reasonably accurate LF tool that can follow the trends of the HF 
one, the resulting error is smooth enough to allow the use of a cheap Radial Basis Function $(\mathrm{RBF})$ model. The objective function $f_{\text {low }}$ is associated with a more challenging design space and as such a Kriging model is being used. For the required tuning of the hyperparameters, ALPSO optimizer from Ref. [38] was used. Finally, based on Eq.10, the HF objective function is estimated by,

$$
\hat{y}(\mathbf{x})=\hat{\mu}+\boldsymbol{\psi}^{T} \mathbf{\Psi}^{-\mathbf{1}}(\mathbf{y}-\mathbf{1})+e(\mathbf{x})
$$

Notice how the prediction is corrected by an estimation of the LF error. This provides a mapping of the Kriging predictor from an approximation of the LF space to an approximation of the HF design space [28].

\subsubsection{Surrogate-Based Optimization}

The use of the Kriging model not only improves the accuracy of the metamodeling approximation, but most importantly, extends the possibilities of the surrogate-based optimization method to be used. Despite the cost increase associated with its training, the benefits for using Kriging in an optimization study that involves expensive analyses are higher. A Gaussian-based approximation allows us to estimate the uncertainty of the model itself. Following this, a metric that includes both the estimated model results and its uncertainty can be constructed. In this approach, the metric used is the Expected Improvement (EI) [35] which is a prediction of how much the function of interest will be improved in each design point examined.

$$
E I=\left(y_{\min }-\hat{y}(\mathbf{x})\right) \Phi\left(\frac{y_{\min }-\hat{y}(\mathbf{x})}{\hat{s}(\mathbf{x})}\right)+\hat{s}(\mathbf{x}) \phi\left(\frac{y_{\min }-\hat{y}(\mathbf{x})}{\hat{s}(\mathbf{x})}\right)
$$

Therefore, now the problem is reduced to maximizing this simple function during the suboptimization process.

The advantage of creating a metamodel for the LF tool and using this surrogate-based expression of EI instead of a LF analysis during suboptimization is twofold:

- For a reasonable number of training data in low/mid dimensionality problems, its total cost (including the training process) is lower than using LF analysis during the suboptimization. This decreases the total cost and allows more design space to be explored during this phase. 
- The EI expression uses information from the whole design space, providing a fast global exploration. A similarly extensive search based on Eq.10, using a LF analysis for $\mathrm{y}_{\text {low }}$ would be inefficient within global SBO.

\subsubsection{Multiobjective Optimizer}

Our implementation can tackle MO problems by formulating a pareto front of maximum EI points [28]. This constitutes a cheap MO suboptimization problem, since the EI is defined simply by an algebraic expression, and as such exploring gradient-free optimizers can still be used efficiently.

In this case, a Multiobjective Particle Swarm Optimization (MOPSO) [30] scheme is used to solve the MO suboptimization problem which in a two objective case becomes:

$$
\mathbf{x}_{\mathbf{1}}{ }^{*}, \mathbf{x}_{\mathbf{2}}{ }^{*}, \cdots, \mathbf{x}_{\mathbf{n}}{ }^{*}=\arg \left(\max _{\mathbf{x} \in D}\left(E I_{1}\right) \text { vs } \max _{\mathbf{x} \in D}\left(E I_{2}\right)\right)
$$

The additional advantage of this approach is that since multiple infill points are identified by the solution of the suboptimization problem, they can be sampled in parallel favouring proper load balancing. This more than compensates for any potential expenses due to the MO search. Such an explicit MO suboptimization process and objective space imrovement is not typical in MO SBO methods [34, 39], however it was observed that it is this explicit formulation that guarantees a wider objective space exploration [40].

155 2.2.5. MF Infill Sampling approach

Once the new design points are defined, the infill analyses take place. In this stage, we take advantage of the error surrogate model information to avoid non-required computational expenses. Configurations to which the LF tool is associated with low error ${ }^{4}$ are analysed only with the LF method. To introduce high fidelity information and improve the design space estimation, we impose both $\mathrm{HF}$ and LF analyses in a predefined iteration frequency.

The described methodology is hereafter referred to as MF EI.

\subsection{Hypervolume Indicator}

The paper aims at comparing two methods for multifidelity optimization. The traditional Pareto front plots show quickly the method strength and weaknesses according

\footnotetext{
${ }^{4}$ Depending on the engineer's needs.
} 


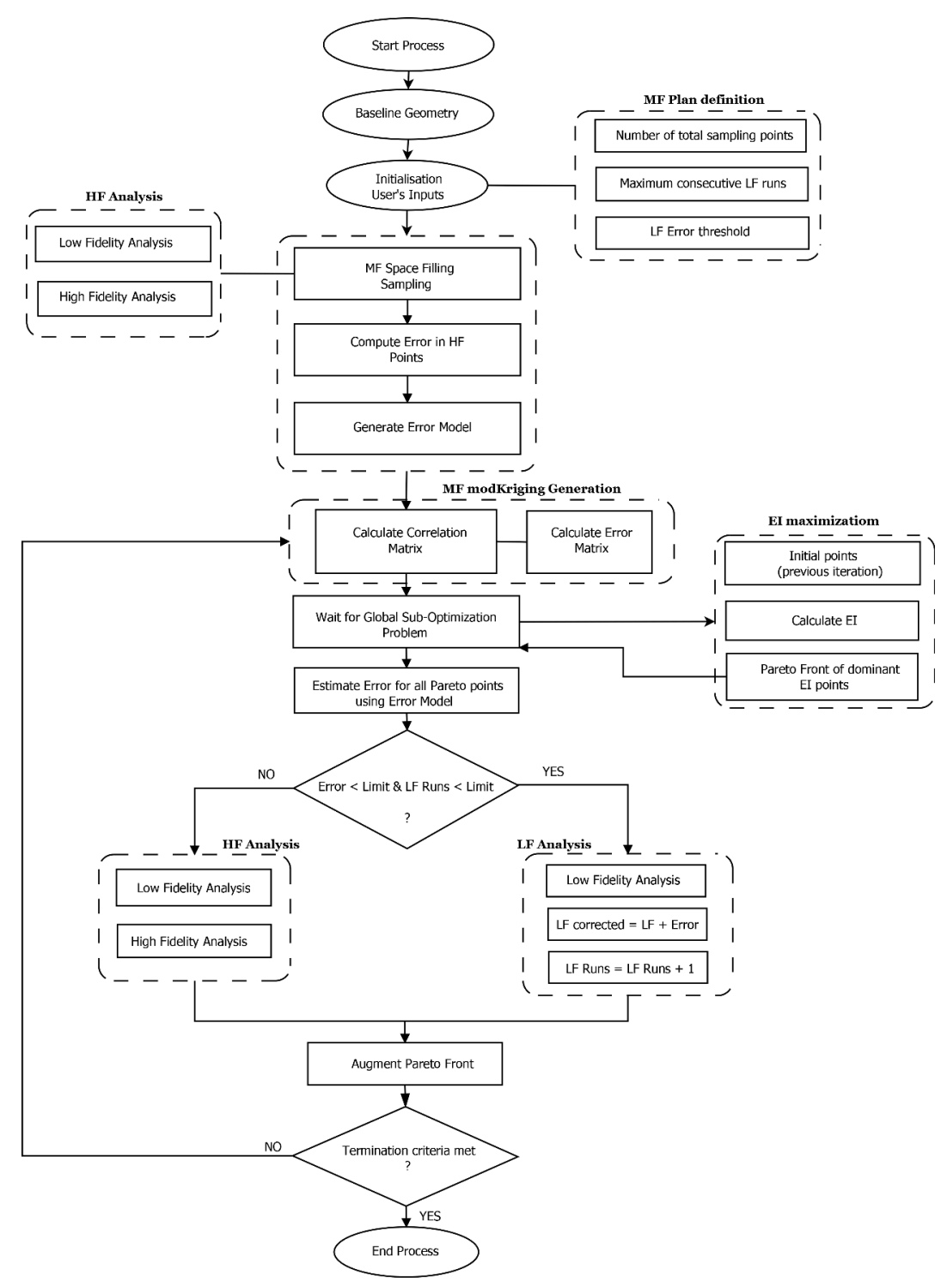

Figure 2: Multiobjective Multifidelity Expected Improvement methodology 


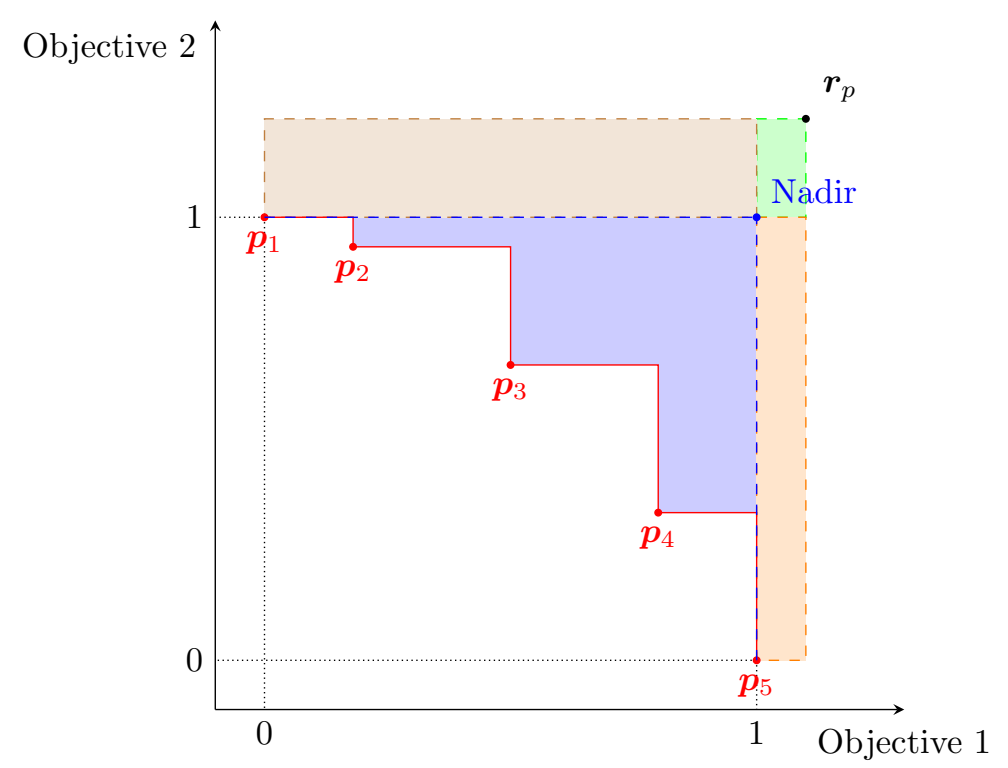

Figure 3: Hypervolume definition from a normalised Pareto Front $\left\{\boldsymbol{p}_{i}, i \in\left[0, \ldots, m_{p}\right]\right\}$ and a reference point $\boldsymbol{r}_{p}$.

165 to the portion of the design space dominated by each method at a particular number of CFD calls. It does not show however, how the Pareto front evolved in the previous steps. The hypervolume indicators [41] aim at answering this question, by computing at any time of the optimisation, the objective space area dominated by a particular front, as shown in figure 3 . To bound the area, a reference point need to be chosen, and depending on its location, the hypervolume indicators will reflect more the domination of the Pareto front (including the number of points making the front) or the extreme points location. It is worth adding that the higher the indicator, the better the method.

In the method comparisons, in section 4.1 for the Garteur test case and section 4.2 for the RAE test case, the hypervolume indicators are used to study the Pareto front convergence, at different stages of the optimisation counted as number of CFD calls. The reference point is chosen close to the further Nadir point.

The hypervolume indicators also include the convergence of what is called the surrogate Pareto front (labelled as Surrogate PF), that is the Pareto front composed of all methods points. It is therefore the best Pareto front that is known to be theoretically achievable if we combine all the methods, without knowing the true function, but no 


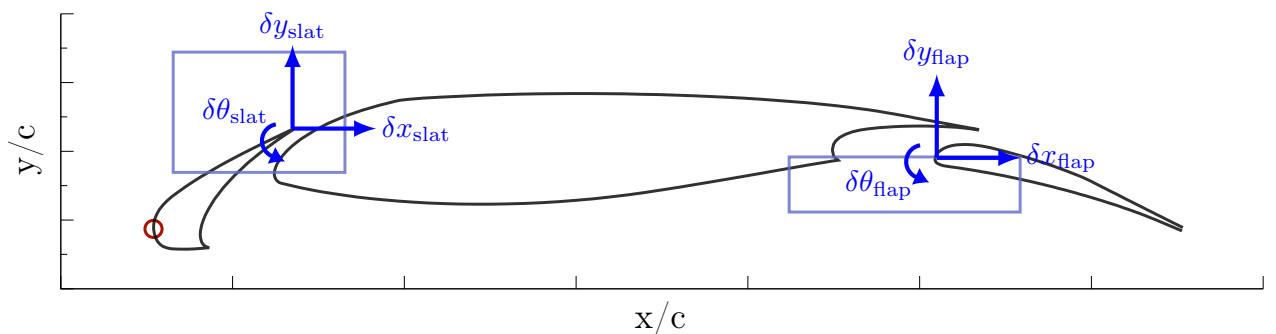

Figure 4: GARTEUR airfoil

method reaches it independently.

\section{Test cases}

\subsection{Garteur test case}

\subsubsection{Problem Formulation and Geometry Parameterization}

The test case presented in Ref. [33] is also used in this study. The geometry is a two-dimensional multielement airfoil composed of a single-slotted slat and a flap. The design variables are shown in figure 4 (oriented in positive movement): the goal of the optimization is to set the slat and flap positions and deflection angles in order to maximize the two-dimensional performance at take-off. The flow conditions [31] are shown in table 1 and the optimization is performed at fixed angle of attack taken as the angle of attack at maximum lift $\alpha_{c l_{\max }}$.

High-lift devices are used for take off and landing, when both lift and drag are critical [15]. In addition to considering the lift generated, drag must also be considered to fulfil the climb index at take off or the proper slowing down of the aircraft when reaching the ground. The optimisation is considering both objectives competing and a multiobjective formulation is used to obtain a Pareto front solution instead of a single compromise optimum. In this way, the objectives are set in equation (14) [42].

$$
f^{(1)}=-\frac{c_{l}}{c_{l_{\text {datum }}}}, \quad f^{(2)}=\frac{c_{d}}{c_{d_{\text {datum }}}}
$$




\begin{tabular}{lll}
\hline \hline Parameters & Notation & Value \\
\hline Reynolds number & $R e$ & $4.10 \cdot 10^{6}$ \\
Mach number & $M$ & 0.20 \\
Fixed Angle Of Attack & $\alpha_{c l_{\max }}$ & $24[\mathrm{deg}]$ \\
\hline Trigger for bad prediction & $\rho_{\mathrm{bad}}$ & 0.0 \\
Trigger for good prediction & $\rho_{\mathrm{good}}$ & 0.0 \\
Trust region shrink value & $\gamma_{s}$ & 2 \\
Trust region expansion value & $\gamma_{e}$ & 2 \\
Number of objectives required & $n_{f}$ & 1 \\
Number of points required & $n_{p}$ & 2 \\
Initial trust region size & $\tau_{0}$ & 6 \\
\hline \hline
\end{tabular}

Table 1: High-Lift optimization parameters

\subsubsection{High Fidelity Analysis Tool}

The commercial tool Fluent [43] is used as the most accurate tool for the study. The mesh is generated with ICEM [44] and a mesh regeneration method is preferred due to the large movement allowed for the slat and flap. Despite being two-dimensional, the geometry is complex as it involves close elements and sharp edges. The mesh is unstructured with a fine density close to the wall and is generated automatically for each new geometries. It uses a wall function to keep the number of cells around 300,000 elements so that the simulation time is compatible with an optimisation. The mesh size was selected with a mesh convergence study in [45] and validated against wind tunnel experiments in [33].

Reynolds Averaged Navier Stokes (RANS) simulations are the workhorse of the industry [46] to obtain accurate but relatively fast aerodynamic performances. The SpalartAllmaras turbulence model is used after comparing experimental and numerical results in Ref. [33] where the authors have reached the same conclusion as in Ref. [47]. An Upwind second-order scheme is used for all equations after iteration 500 to secure convergence robustness in the start of the simulation. The flow is considered converged when residuals 
fall below $10^{-5}$, or if the number of iterations is greater than $2000^{5}$. Because convergence is not always achieved, the lift and drag coefficients are averaged over the last 100 iterations. A single point evaluation requires between 10 to 20 minutes to run, forming the expensive function in our study. Validation against wind tunnel data is performed in Ref. [33].

\subsubsection{Low-Fidelity Analysis Tool}

The MSES software from Professor Drela, MIT is used as the low-fidelity estimator. It is a coupled viscid/inviscid software: the inviscid Euler equations are coupled with a multiequation integral formulation, the coupling being performed with a Newton solver. This software handles wakes from each elements and can predict separation in the cove regions. It allows a good prediction of the lift and drag even near maximum lift [48].

When used in challenging aerodynamic case, such as high-lift conditions, a single point evaluation requires up to 2 minutes. An extensive separation region, that might appear in a bad design generated during the optimization process, would lead to divergence in the coupling procedure. This phenomena is amplified by the quality of the discretization used for the Euler solver. Despite the attention given to the selection of the mesh points distribution on the profile, divergence occurs for bad designs resulting in a restricted design space.

\subsection{RAE2822 test case}

\section{3.2.1. Problem formulation}

The RAE 2822 airfoil provides a popular test case among researchers, as it resembles a typical industrial transonic airfoil design study. The respective physical conditions, which are shown in Table 2, correspond to actual aircraft operating conditions.

As in the Garteur case, this problem is also set up in an explicit MO formulation by using Lift and Drag as the functions of interest. These are provided in a coefficient form as follows:

$$
f_{1}=-C_{l}, f_{2}=C_{d}
$$

\footnotetext{
${ }^{5}$ This may be not enough to have fully converged flow but is sufficient to capture most of the flow physics
} 
Such a MO design problem formulation is an industrial standard for several reasons.

It provides more information than a corresponding $\mathrm{SO}$ one, in a cost-effective manner. Several optimum configurations are readily available in the engineer's disposal and the pareto front can act as a potential baseline for later-stage local and more detailed tradeoff studies. The existence of such a tool is highly beneficial as it supports decision making in a more reliable way.

\subsubsection{Geometry parameterization}

For the parameterization of this shape design problem, the method of Free Form Deformation (FFD) [49] was used with the displacements of the control points, defining the shape deformation in the $\mathrm{x}$ and $\mathrm{y}$ direction. In a shape design problem, the bound values are extracted by the design requirements and are unique for each case. As such, our flexible implementation allows the explicit definition of bounds. To simulate an industrial application with increased complexity, 8 active control points were used, creating a 16-D design space.

Table 2: Physical conditions for RAE2822 case

\begin{tabular}{lrl}
\hline \hline Condition & Value & Units \\
\hline Angle of Attack & 2.31 & deg \\
Mach Number & 0.729 & - \\
Reynolds number & $6.510^{6}$ & \\
Pressure & 108987 & $\mathrm{~Pa}$ \\
Temperature & 255.55 & $\mathrm{~K}$
\end{tabular}

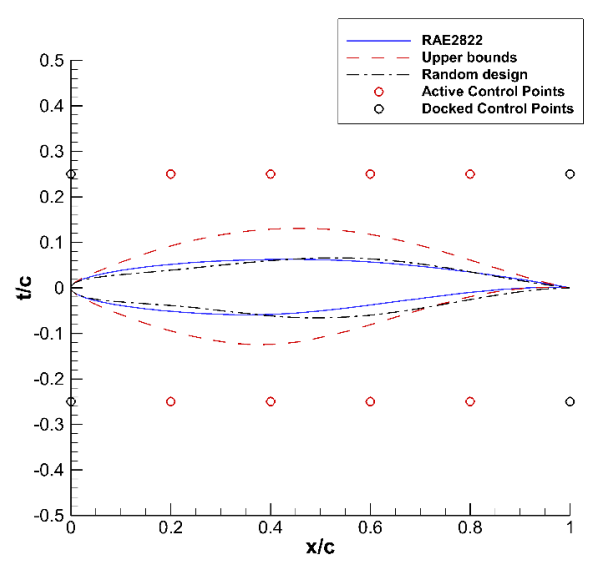

Figure 5: FFD control points around the RAE 2822 Airfoil in this design problem.

\subsubsection{High Fidelity Analysis Tool}

The HF analyses are performed using the commercial solver ANSYS Fluent [43], to provide the global aerodynamic coefficients. Since RANS equations are solved, a 
structured C-type grid with a $y^{+} \approx 1$ is used. A grid validation [50] study showed that a grid of 38000 quad cells was sufficiently accurate. In this problem, grid renegeration was used instead of grid deformation, since the computational cost is low. We exercised special care to ensure that grid regeneration would be robust to geometry changes that arise throughout the optimization process. An implicit density-based Roe-FDS solver with a $2^{\text {nd }}$ order upwind discretization scheme was used and Sutherland's model for the dynamic viscosity was employed. In this test case, turbulence effects were modelled using $k-\omega$ SST [51], as to the authors' experience it provides the most accurate results for attached slender body transonic aerodynamic flows. It also forms a robust solution, in contrast to when being used in cases like high-lift multi-element GARTEUR airfoil which involve partially or extensively separated flow. The maximum number of iterations for convergence was set to 3000 . To ensure robustness, in cases where stable limited oscillations are present in the solution, the final result was averaged using values from the last 50 iterations.

\subsubsection{Low Fidelity Analysis Tool}

The cost and accuracy of the LF tool is crucial for the success of any MF method. In this work, we are using VGK [52], a viscosity corrected numerical tool based on the method of Garabedian and Korn [53]. Although accurate around its calibration range, it is not considered robust when used within a wide design space. This is a direct result of the physics, involving flow separation regions or strong shocks.

\section{Results}

The MF TR and EI methodologies examined in this paper are compared against a single fidelity SBO HF EI approach (labelled as HF EI) and a gradient-free optimization calling directly the HF tool without the use of a surrogate model (labelled as TS-only HF or MOPSO-only HF depending on the optimizer used - one for each test $\operatorname{case}^{6}$ ). For the latter, the Tabu Search optimizer is used in the Garteur case and our inhouse implementation of MOPSO is used in the RAE case. Therefore, apart from a comparison

\footnotetext{
${ }^{6}$ When the optimizer is used directly on the analysis - like the TS/MOPSO only HF Cases - the hyperbolic indicator is not defined, so it is not included in the corresponding figures
} 
between two popular MF methods, we also intent a comparison against direct gradientfree optimization and single fidelity global SBO technique.

\subsection{Garteur}

Figure 6 shows the hypervolume indicators convergence, introduced in section 2.3, as well as the Pareto front after about $125 \mathrm{HF} \mathrm{CFD}^{7}$ calls for each method.

The Tabu Search optimizer, acting directly on the HF analyses, finds good compromise points in a few iterations. However, as it can be seen on Figure 6 the Pareto front is narrow since TS first works locally and then extends its Pareto front. In the following iterations the front becomes wider, as implied by the hypervolume indicator convergence. This, however, requires many more HF calls.

Following a brief initial sampling (6 high-fidelity and 18 low-fidelity), MF EI is very quick to discover the Pareto front thanks to the problem's low dimensionality. In the initial iterations, EI being space relatively sparse, the explorative attribute of the method leads to a high infill success rate, as it is easy to find even slight improvements throughout the whole design space. However, after only 40 CFD calls the method seems to converge and no further improvement occurs. In fact, the method becomes inferior to the exploitative TR-based approach after $100 \mathrm{HF}$ calls $-50 \%$ of the eventual total allocated computational budget. This is again a direct result of the method behavior: with Pareto points already identified, the —now multimodal EI suboptimization problem- becomes more challenging and the method keeps exploring the design space for new design trends to find improvements. It is important to note that the low-fidelity tool, MSES, is robust in specific design space location, but when used as part of an explorative optimizer, the lack of robustness complicate the task. The lack of pure exploitation and local search near the current Pareto points, combined with the LF tool inability to converge in all of the design space, leads to this early convergence. The former is an inherent attribute of the methodology, but the latter is a problem already observed in other applications of such methods [25], and it is briefly discussed below.

It is important to discuss the effect that a LF tool has on the exploration MF methodologies convergence, especially in the presence of a wide design space. The general effect

\footnotetext{
${ }^{7}$ Depending on optimizer implementation
} 
of MSES is not beneficial for the widening of the Pareto front. This is mostly observed in extreme operational regions like high $C_{l}$ regions, that include high interactions between wakes and large flow separation, complicating the coupling procedure between Euler inviscid and viscous boundary later solutions. Hence, the initial global sampling does not provide information on an extensive design space region, and the MF EI method fails to exploit its exploration attributes. As such, the use of a tool which is a not robust in the whole design space is not appropriate for design space exploration even in the conceptual design stage, especially if the engineer is interested in finding novel design trends. In such cases, using information from a more mature stage materialised by higher fidelity tools is preferable and a HF SBO method is definitely superior. Nevertheless, in regions where MSES is robust and accurate, the MF optimization process is more efficient: it provides a more optimal Pareto front. Following this, it is not a complete surprise that MF TR provides, in a small extend, a denser (almost 3 times as dense compared to the MF EI one) and wider Pareto front. The MF TR Pareto front convergence is initially slower due to the lack of sampling which limits the source of information MSES. The LF tool provides useful design trends but a couple of iterations are required before these are translated to dominant points. These uncorrected inaccuracies restricts the MR TR to the same convergence behaviour as TS only on HF. Nevertheless, the convergence is consistent and explores more of the design space, in a similar manner as TS only on HF, but with a quicker Pareto front extension. MF TR is able to locate design points which are better than the ones discovered by HF EI, in the whole range of its Pareto front. Furthermore, it exhibits a slightly wider Pareto front, especially in the low drag region. The better MF TR hypervolume indicator, compared to the TS only on HF, is explained by the extended objective space exploration. Despite this, the points predicted by TS only on $\mathrm{HF}$ in the mid $C_{l}$ regime are dominating the respective ones from all other methods. MF EI provides an improvement over the results from the rest of the methods in the high $C_{l}$ region, in the order of $\approx 7-12 \%$. In lower lift design points, its performance is similar to MF TR, while it is being inferior in the lowest $C_{l}$ objective space. Despite this fact, the use of MF data in the EI method can be considered as beneficial, since only one MF EI Pareto point is dominated by HF EI. The drawback of the former though is the reduced number of Pareto front points to around $30 \%$ of the HF EI pareto points. 


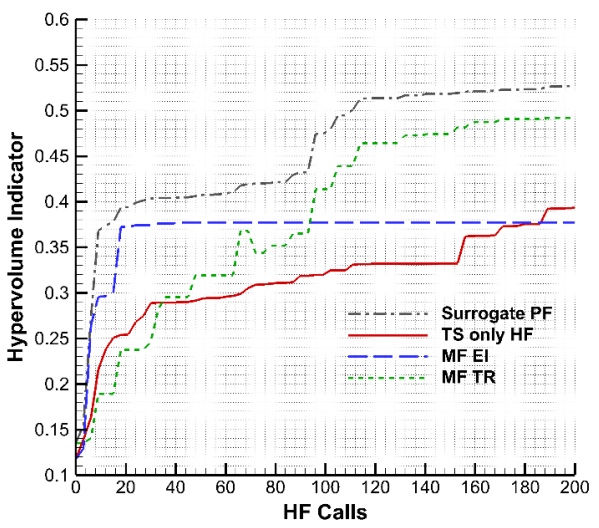

(a) Hypervolume indicators

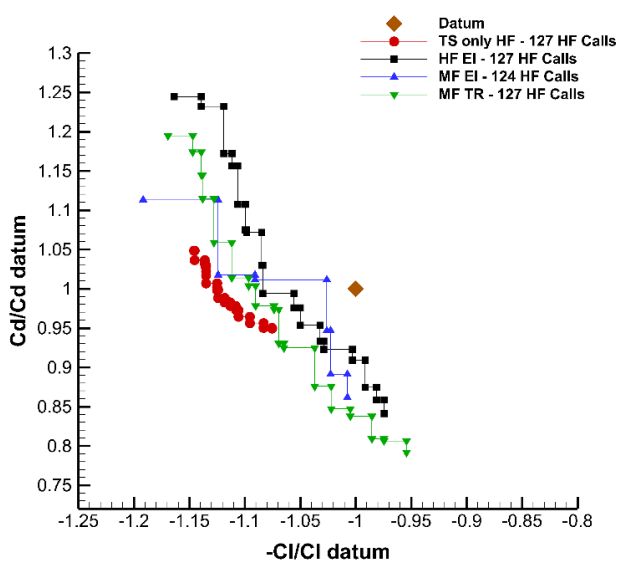

(b) Pareto front

Figure 6: Hypervolume indicator convergence and Pareto front after 125 CFD calls for the Garteur case.

The above conclusions are better supported by a parallel coordinate plot [54], shown in Figure 7. It shows the difference in the design variable values and design trends behind each methods' Pareto points. As previously, the Pareto front points are displayed at the same number of HF calls. The following information can be extracted:

- A qualitatively consistent slat movement is observed: all methods identify the importance of moving the slat forwards and down to increase wake interaction from slat to the main airfoil element.

- Discrepancies in slat angle: there is moderate movement from all methods but MF EI tends to deflect the slat as much as possible for increasing the lift. These discrepancies arise from the difference in exploration capabilities between the methods. It is no consequence that it is the explorative MF EI method that takes advantage of the positive slat rotation trend, maximising its benefit by generating max slat angle high-lift design. The There is also an agreement in the low-drag region, with lower slat deflection due to lower flap deflection.

- Low-drag points have different arrangement compared to datum: they use a lower flap position with a reduced deflection: less energy is extracted, with flap deflection being less affected from the main element to keep flap boundary layer attached. 


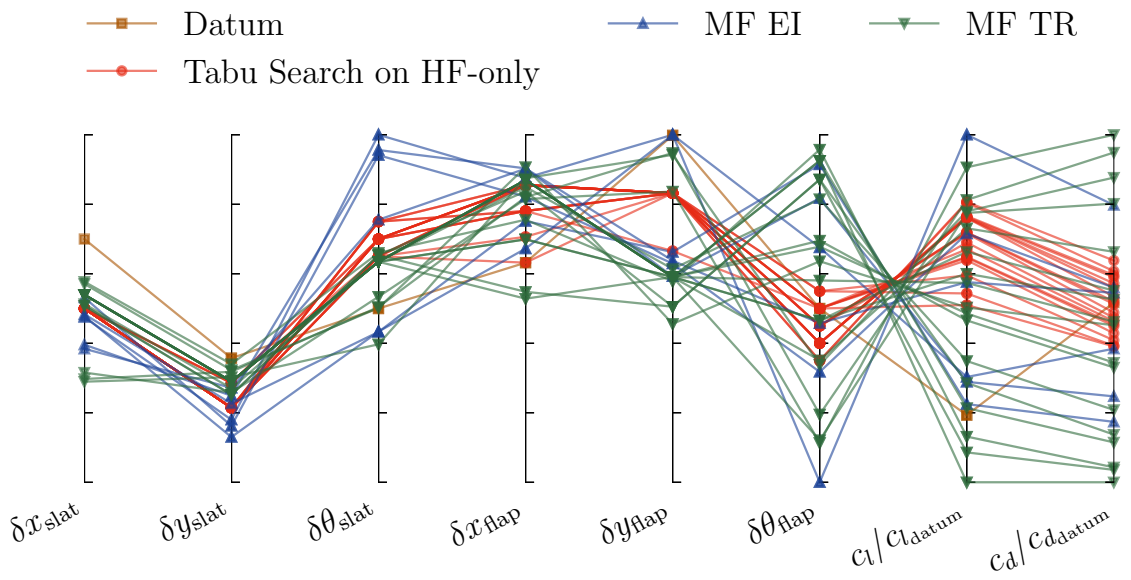

Figure 7: Parallel-coordinate plot for the Garteur case Pareto points after 125 CFD calls.

- Only the MF methods extensively explores the optimal design space, thanks to the quick identification and exploitation of the flap deflection. With this variable being the most dominant, MF EI uses the maximum allowed deflection to get maximum lift.

- The HF and MF methods' points tend to follow the same arrangement. Therefore, the discrepancies from the MSES LF tool are well corrected. MF approaches are quicker in finding promising regions. TS only on HF is the most efficient method at exploiting that is to find the best solutions in the vicinity of datum point.

To conclude this comparison performed on the Garteur case, the TS only on HF is efficient in exploiting locally near the datum, but requires more HF calls than the rest of the methods to expand the Pareto front. The HF EI explores design space, but lacks exploitation and its points are dominated by the other methods. The MF TR has a behaviour very similar to HF EI, but shows a quicker Pareto front advancement. The MF EI approach has fewer Pareto points than all the other case, but exploits the high-lift mechanisms better due to its initial full design space exploration. The design changes of the SBO methods are consistent with the direct gradient-free optimization. In regards to the MF methods, the LF tool inaccuracies have only a minimum effect in the final Pareto front values. 


\subsection{RAE2822}

In the initial HF infill calls, MF TR develops a Pareto front faster. However, following the end of the initial sampling, MF EI starts exploiting the surrogate creating

EI dominates MF TR. An investigation of the Pareto front reveals that this domination originates from the high- $C_{l}$ region.

By comparing the MF TR to the MF EI approach in terms of final Pareto front, it is evident that both methods exhibit similar objective space exploration as they both provide a wide Pareto front. The similarities continue in the objective function values of the mid and low $C_{l}$ regions. However, in the high $C_{l}$ objective space MF EI shows an improved performance that roughly translates to $27 \%$ drag decrease and $15 \%$ lift increase.

The use of variable fidelity tools within the EI method is also assessed by comparing the HF EI method against the MF EI method. There is a definite correlation between HF EI and MF EI Pareto front as the corresponding values are very close across the whole Pareto front. This shows that the LF tool provides correct design trends in a wide design space range. Therefore, the error correction is not only accurate, but also efficient as the quick RBF correction is sufficient in steering towards HF optimality. However, the computational cost between the three SBO methods is similar, with only exploration being slightly improved in the MF EI case. Hence, it can be concluded that the benefits associated with using MF SBO methods for this design scenario are small.

The direct optimization that uses our MOPSO implementation, develops a dense but narrow Pareto front. This is important to stress out since the purpose of a MO formulation is to explore different design possibilities in a wide objective regime. Although not shown here, no significant improvements are observed in the Pareto front width or domination at later stages of the optimization: the swarm does not find new Pareto dominant points. MOPSO HF results are almost completely dominated by the MF and HF EI ones. However, the tradeoff region includes some designs developed by MOPSO which are still superior to the ones identified by HF EI and MF EI. The MF TR configurations however are mainly dominated by MOPSO in the high-lift anchor point region albeit MF TR Pareto front extends more in the high-lift region. Overall, it can be stated that SBO 
increases the design space exploration for a similar cost.

An example of the Pareto front airfoil shapes is given in Figure 9. Despite the similar Pareto points between MF EI and HF EI showing proximity on the objective space, the respective shapes are distinctively different, exploring different points in the design space. However, MF TR and MOPSO approaches are more similar: the former methods are global whereas the latter ones are more local. Therefore MF TR and HF MOPSO dominate in area close to datum (in design space) but HF EI and MF EI explore configurations different from datum. By specifically examining the high $C_{l}$ shapes, it is evident that these are very different from the datum, requiring global exploration to find them.

The MF EI method generates the high lift from its aft camber design as shown in 9 . Additionally, it is significantly thinner than the one designed by the MF TR approach in order to compensate for the wave drag introduced due to the achieved lift. In the compromise region all geometries exhibit a significant thickness reductionl the exception being the airfoil resulting from MOPSO, since it is only slightly thinner (mainly from the upper surface) than the datum while maintaining the original RAE2822 shape. The same is not valid for the configurations by the MF TR and MF EI approaches which introduce a positive camber in the area before the mid chord. Evidently, the MF TR methodology retains the supercritical shape while producing the necessary lift through an aft camber design. A corresponding configuration is also defined by MF EI with its distinctive characteristic being the absence of an aft camber and an earlier thickening than the one by MF TR. The low drag MF designs both feature an inverse aft camber, with the MF TR being thinner and displaying a positive camber downstream of the LE area. It is evident that these designs are shaped as they are because of the positive angle of attack used in this optimisation problem (they minimise drag by reducing their thickness and creating zero lift). For an angle of attack of zero they would obviously produce negative lift. 


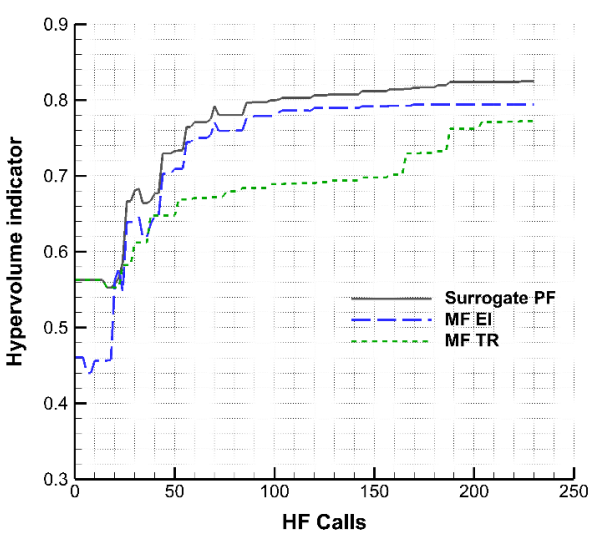

(a) Hypervolume indicators

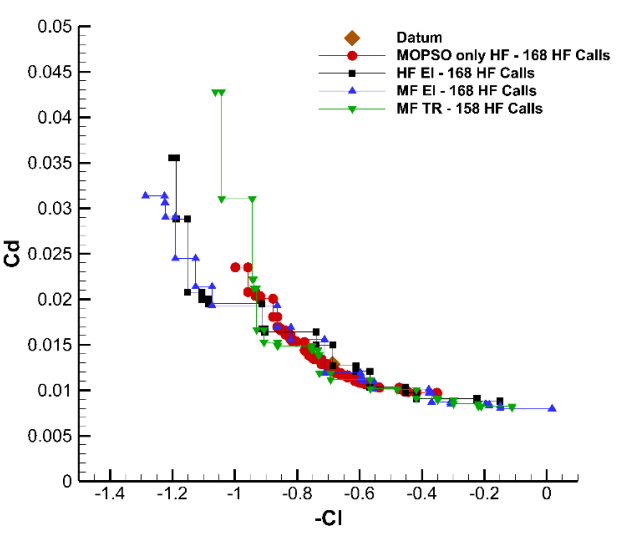

(b) Pareto front

Figure 8: Hypervolume indicator convergence and Pareto front after 125 CFD calls for the RAE case.

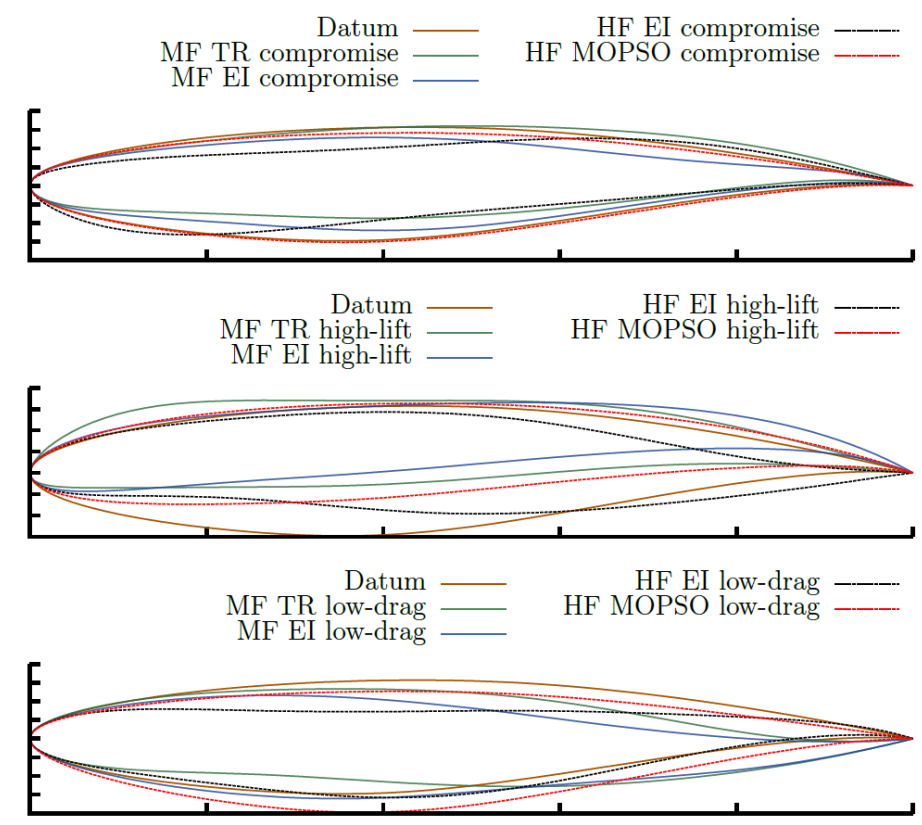

Figure 9: Three representative Pareto front shapes for the MF TR and MF EI methods. 


\section{Conclusion}

Optimization techniques. An exploitation-based Multifidelity Trust Region framework is compared against a more explorative Multifidelity Expected Improvement methodology. The methods are also assessed by comparing them against benchmark gradient-free optimization tools such as Tabu Search and the Multi Objective Particle Swarm Opti-

\section{Acknowledgments}

This project was co-funded by Innovate UK as part of the ATI Agile Wing Integra-

tion Project and ATI Enhanced Fidelity Transonic Wing Project, both led by Airbus. 
The authors would like to thank Professor Mark Drela (MIT) for providing the MSES software.

\section{References}

[1] J. Wild, Multi-objective constrained optimisation in aerodynamic design of high-lift systems, International Journal of Computational Fluid Dynamics 22 (3) (2008) 153-168. doi:10.1080/ 10618560701868420.

[2] N. Ali, K. Behdinan, Optimal geometrical design of aircraft using genetic algorithms, Article in Transactions - Canadian Society for mechanical Engineering 26 (4) (2003) 373-388.

[3] R. Hilbert, G. Janiga, R. Baron, D. Thevenin, Multi-objective shape optimization of a heat exchanger using parallel genetic algorithms, International Journal of Heat and Mass Transfer 49 (1516) (2006). doi:10.1016/j.ijheatmasstransfer.2005.12.015.

[4] A. Amrit, L. Leifsson, S. Koziel, Multi-fidelity aerodynamic design trade-off exploration using pointby-point pareto set identification, Aerospace Science and Technology 79 (2018). doi:10.1016/j. ast. 2018.05 .023 .

[5] R. P. Liem, G. K. W. Kenway, J. R. R. A. Martins, Multimission airfraft fuel-burn minimization via multipoint aerostructural optimization, AIAA Journal 53 (1) (2015) 104-122. doi:10.2514/1. J052940.

[6] T. Kipouros, M. Molinari, W. N. Dawes, G. T. Parks, M. Savill, K. W. Jenkins, An investigation of the potential for enhancing the computational turbomachinery design cycle using surrogate models and high performance parallelisation, in: Proceedings of ASME Turbo Expo, Vol. 6, ASME, Montreal, Canada, 2007, pp. 1415-1424, paper GT2007-28106. doi:10.1115/GT2007-28106.

[7] P. A. LeGresley, J. J. Alonso, Airfoil design optimization using reduced order models based on proper orthogonal decomposition, in: FLUIDS 2000 Conference and Exhibit, AIAA Paper, 2000, p. 2545 .

[8] N. Bartoli, T. Lefebvre, S. Dubreui, R. Olivanti, R. Priem, M. Bons, N., J.R.R.A., J. Morlier, Adaptive modeling strategy for constrained global optimization with application to aerodynamic wing design, Aerospace Science and Technology 90 (2019) 85-102. doi:10.1016/j.ast.2019.03. 041.

[9] T. W. Simpson, T. M. Mauery, J. J. Korte, F. Mistree, Kriging models for global approximation in simulation-based multidisciplinary design optimization, AIAA Journal 39 (12) (2001). doi: $10.2514 / 2.1234$

[10] J. M. Parr, A. J. Keane, A. I. J. Forrester, C. Holden, Infill sampling criteria for surrogate-based optimization with constraint handling engineering optimization, Engineering Optimization 44 (10) (2012). doi:10.1080/0305215X.2011.637556.

[11] G. Giangaspero, D. MacManus, I. Goulos, Surrogate models for the prediction of the aerodynamic performance of exhaust systems, Aerospace Science and Technology 92 (2019) 77-90. doi:10.1016/ j.ast.2019.05.027. 
[12] A. Batrakov, A. Kusyumov, S. Mikhailov, G. Barakos, Aerodynamic optimization of helicopter rear fuselage, Aerospace Science and Technology 77 (2018) 704-712.

[13] A. A. Mullur, A. Messac, Extended radial basis functions: More flexible and effective metamodeling, AIAA Journal 43 (6) (2005). doi:10.2514/1.11292.

[14] R. A. Jacobs, M. I. Jordan, S. J. Nowlan, G. E. Hinton, Adaptive mixtures of local experts, Journal Neural Computation 3 (1) (1991) 79-87. doi:10.1162/neco.1991.3.1.79.

[15] A. Flaig, R. Hilbilg, High-lift design for large civil aircraft, in: High Lift System Aerodynamics, AGARD CP-515, 1991, pp. 31-1-31-12.

[16] A. J. Keane, Cokriging for robust design optimization, AIAA Journal 50 (11) (2012) 2351-2364. doi:10.2514/1. J051391.

[17] J. W. Bandler, R. Biernacki, S. H. Chen, P. A. Grobelny, R. H. Hemmers, Space mapping technique for electromagnetic optimization, IEEE Transactions on Microwave Theory and Techniques 42 (12) (1994) 2536-2544. doi:10.1109/22.339794.

[18] J. P. Jarrett, T. Ghisu, An approach to multi-fidelity optimization of aeroengine compression systems, in: $12^{\text {th }}$ AIAA Aviation Technology, Integration, and Operations (ATIO) Conference and $14^{\text {th }}$ AIAA/ISSMO Multidisciplinary Analysis and Optimization Conference, AIAA, Indianapolis, USA, 2012, paper 2012-5634. doi:10.2514/6.2012-5634.

[19] N. M. Alexandrov, R. M. Lewis, C. R. Gumbert, L. L. Green, P. A. Newman, Approximation and model management in aerodynamic optimization with variable-fidelity models, Journal of Aircraft 38 (6) (2001) 1093-1101. doi:10.2514/2.2877.

[20] N. M. Alexandrov, J. E. Dennis, R. M. Lewis, V. Torczon, A trust-region framework for managing the use of approximation models in optimization, Structural optimization 15 (1) (1998) 16-23. doi:10.1007/BF01197433.

[21] S. Choi, J. J. Alonso, I. M. Kroo, M. Wintzer, Multifidelity design optimization of low-boom supersonic jets, Journal of Aircraft 45 (1) (2008) 106-118. doi:10.2514/1.28948.

[22] H. S. Chung, J. J. Alonso, Design of a low-boom supersonic business jet using cokriging approximation models, in: $9^{\text {th }}$ AIAA/ISSMO Symposium on Multidisciplinary Analysis and Optimization, AIAA Paper, 2002. doi:10.2514/6.2002-5598.

[23] A. Ariyarit, M. Sugiura, Y. Tanabe, M. Kanazaki, Hybrid surrogate-model-based multi-fidelity efficient global optimization applied to helicopter blade design, Engineering Optimization 50 (6) (2018) 1016-1040.

[24] A. Ariyarit, M. Kanazaki, Multi-fidelity multi-objective efficient global optimization applied to airfoil design problems, Applied Sciences 7 (12) (2017) 1318.

[25] J. Demange, M. Savill, T. Kipouros, A multifidelity multiobjective optimization framework for high-lift airfoils, in: $17^{\text {th }}$ AIAA/ISSMO Multidisciplinary Analysis and Optimization Conference, AIAA, Washington, USA, 2016, p. 3367, paper 2016-3367. doi:10.2514/6.2016-3367.

[26] A. R. Conn, K. Scheinberg, L. N. Vicente, Global convergence of general derivative-free trust-region algorithms to first and second-order critical points, SIAM Journal on Optimization 20 (1) (2009) 387-415. doi:10.1137/060673424. 
[27] A. March, K. Willcox, Provably convergent multifidelity optimization algorithm not requiring highfidelity derivatives, AIAA Journal 50 (5) (2012) 1079-1089. doi:10.2514/1. J051125.

[28] S. G. Kontogiannis, A. M. Savill, T. Kipouros, A multi-objective multi-fidelity framework for global optimization, in: 58th AIAA/ASCE/AHS/ASC Structures, Structural Dynamics, and Materials Conference, 2017, p. 0136.

[29] D. R. Jones, A taxonomy of global optimization methods based on response surfaces, Journal of Global Optimization 21 (2001) 345-383. doi:10.1023/A:1012771025575.

[30] J. E. Alvarez-Benitez, R. M. Everson, J. E. Fieldsend, A Mopso Algorithm Based Exclusively on Pareto Dominance Concepts, Springer Berlin Heidelberg, Berlin, Heidelberg, 2005, pp. 459-473. doi : 10.1007/978-3-540-31880-4_32.

[31] J. J. Thibert, The garteur high lift research programme, in: High Lift System Aerodynamics, AGARD CP-515, 1991, pp. 16-1-16-21.

[32] X. He, J. Li, C. A. Mader, A. Yildirim, J. R. Martins, Robust aerodynamic shape optimization from a circle to an airfoil, Aerospace Science and Technology 87 (2019) 48-61.

[33] J. Demange, M. Savill, T. Kipouros, Multifidelity optimization for high-lift airfoils, in: $54^{\text {th }}$ AIAA Aerospace Sciences Meeting, AIAA, San Diego, USA, 2016, p. 0557, paper 2016-0557. doi:10. 2514/6.2016-0557.

[34] A. I. Forrester, A. J. Keane, Recent advances in surrogate-based optimization, Progress in Aerospace Sciences 45 (1-3) (2009) 50-79.

[35] A. Forrester, A. Sobester, A. Keane, Engineering Design Via Surrogate Modelling: A Practical Guide, Wiley, 2008.

[36] F. Glover, M. Laguna, Tabu search, Tech. rep., Kluwer Academic Publishers, Boston, MA (1997).

[37] D. M. Jaeggi, G. T. Parks, T. Kipouros, P. J. Clarkson, The development of a multi-objective tabu search algorithm for continuous optimisation problems, European Journal of Operational Research 185 (3) (2008) 1192-1212. doi:10.1016/j.ejor.2006.06.048.

[38] R. E. Perez, P. W. Jansen, J. R. R. A. Martins, PyOpt: A Python-based object-oriented framework for nonlinear constrained optimization, Structures and Multidisciplinary Optimization 45 (1) (2012) 101-118. doi:10.1007/s00158-011-0666-3.

[39] I. Couckuyt, D. Deschrijver, T. Dhaene, Fast calculation of multiobjective probability of improvement and expected improvement criteria for pareto optimization, Journal of Global Optimization 60 (3) (2014) 575-594.

[40] S. G. Kontogiannis, Conceptual multidisciplinary design via a multiobjective multifidelity optimisation method, Ph.D. thesis, Cranfield University (2018).

[41] Y. Cao, B. J. Smucker, T. J. Robinson, On using the hypervolume indicator to compare pareto fronts: Applications to multi-criteria optimal experimental design, Journal of Statistical Planning and Inference 160 (2015) 60-74. doi:10.1016/j.jspi.2014.12.004.

[42] G. Trapani, The design of high-lift aircraft configurations through muti-objective optimisation, Ph.D. thesis, Cranfield University (Mar. 2014).

485 [43] ANSYS, Fluent 14.5, User's Manual, Lebanon, 2013. 
[44] ANSYS, ICEM CFD 14.5, User's Manual, Lebanon, 2013.

[45] J. Demange, Multifidelity multiobjective trust-region-based optimisation for high-lift devices, Ph.D. thesis, Cranfield University (2018).

[46] C. L. Rumsey, S. X. Ying, Prediction of high lift: Review of present CFD capability, Progress in Aerospace Sciences 38 (2) (2002) 145-180. doi:10.1016/S0376-0421(02)00003-9.

[47] J. Wild, Multi-objective constrained optimisation in aerodynamic design of high-lift systems, International Journal of Computational Fluid Dynamics 22 (3) (2008) 153-168. doi:10.1080/ 10618560701868420

[48] C. P. van Dam, The aerodynamic design of multi-element high-lift systems for transport airplanes, Progress in Aerospace Sciences 38 (2) (2002) 101-144. doi:10.1016/S0376-0421(02)00002-7.

[49] T. W. Sederberg, S. R. Parry, Free-form deformation of solid geometric models, in: ACM Siggraph Computer Graphics, Vol. 20, ACM, 1986, pp. 151-160.

[50] P. J. Roache, Verification and validation in computational science and engineering, 1998.

[51] D. C. Wilcox, Turbulence Modeling for Cfd, Vol. 2, DCW industries La Canada, CA, 1998.

[52] D. J. Mitchell, VGK method for two-dimensional aerofoil sections, Part 1: principles and results, ESDU International, 2004.

[53] P. R. Garabedian, D. Korn, Analysis of transonic aerofoils, Comm. Pure and Appl. Maths XXIV (1971) 841-851.

[54] A. Inselberg, B. Dimsdale, Parallel coordinates, in: Human-Machine Interactive Systems, Springer, 1991, pp. 199-233. 
2019-11-29

\section{A comparison study of two multifidelity methods for aerodynamic optimization}

Kontogiannis, Spyridon G.

Elsevier

Kontogiannis SG, Demange J, Savill M, et al., (2020) A comparison study of two multifidelity methods for aerodynamic optimization. Aerospace Science and Technology, Volume 97,

February 2020, Article number 105592

https://doi.org/10.1016/j.ast.2019.105592

Downloaded from Cranfield Library Services E-Repository 